\title{
SOME MEASUREMENTS OF THE INFRARED SPECTRAL RADIANCES AND EMISSIVITIES OF HYDROGEN FLUORIDE IN COMBUSTION GASES AT $1000^{\circ} \mathrm{C}^{*}$
}

\author{
FREDERICK S. SIMMONS \\ Institute of Science and Technology, University of Michigan
}

(Received 30 June 1963)

\begin{abstract}
Spectral radiances and emissivities were determined from moderate-resolution emission and absorption measurements in the exhaust gases of a small rocket motor. Data was obtained in the 2 to $3 \mu$ region for $\mathrm{ClF}_{3}-\mathrm{NH}_{3}$ combustion products in which hydrogen fluoride was the principal infrared emitting species. The results are presented as average spectral emissivities, which are considered to be representative of $3.0 \mathrm{~cm}$-atm of $\mathrm{HF}$ at $1250^{\circ} \mathrm{K}$. The experimental techniques and methods of data analysis are discussed in some detail.
\end{abstract}

SPECTRAL emissivities in the infrared vibration-rotation bands of combustion gases are required for a number of engineering purposes, such as calculations of radiant heat transfer and studies of combustion dynamics in propulsion devices. The combustion products of most importance in this regard are $\mathrm{CO}_{2}, \mathrm{H}_{2} \mathrm{O}$, and $\mathrm{HF}$. Although the infrared spectra of these molecules have been known for a great many years, only recently has attention been given to the determination of quantitative spectral emissive properties, especially at elevated temperatures. Carbon dioxide has received most of this attention; measurements of spectral emissivity in the 2.7 and $4.3 \mu$ bands have been made in a furnace-heated cell, ${ }^{(1,2,3)}$ a shock tube, ${ }^{(4)}$ and a small rocket motor. ${ }^{(5)}$ The resultant data, representative of a wide range of optical depths, can be well correlated by use of a statistical band $\operatorname{model}^{(3)}$ and are in reasonable agreement with approximate theoretical calculations. ${ }^{\left({ }^{6}\right)}$ Similar measurements of spectral emissivity have been made and correlations are being sought for the 2.7 band of water vapor at elevated temperatures. $(7,8,9,10)$ On the other hand, except for a recent determination of total emissivity in an ammoniafluorine flame ${ }^{(11)}$, no high-temperature experimental data for hydrogen fluoride have been reported in the literature.

The present work consists of a set of emission and absorption measurements obtained at moderate resolution in the combustion products of liquid ammonia and chlorine trifluoride, a mixture of $\mathrm{HF}, \mathrm{HCl}$, and $\mathrm{N}_{2}$, at a temperature of about $1250^{\circ} \mathrm{K}$. Spectral radiances and emissivities were obtained at spectral slit widths of 0.005 and $0.02 \mu$ in the 2 to $3 \mu$ and the 2 to $5 \mu$ regions respectively.

* The experimental measurements reported herein were performed at Rocketdyne, a division of North American Aviation, Inc., Canoga Park, California, during 1961. The work was supported by the Advanced Research Projects Agency, Department of Defense, in part through the Air Force Cambridge Research Laboratory under contract AF 19(604)-5701, and in part through the Air Force Space Systems Division under contract AF 04(647)-644, via General Dynamics/Astronautics, San Diego, California. 


\section{EXHAUST GAS PROPERTIES}

The absorption and emission measurements to be reported were made near the nozzle exit of a small water-cooled liquid-propellant rocket thrust chamber. The $\mathrm{ClF}_{3}-\mathrm{NH}_{3}$ propellant combination was selected as a convenient means for producing hydrogen fluoride as the principal infrared emitting species in the exhaust. Typical values of the exhaust composition predicted from the standard thermochemical relations ${ }^{(12)}$ on the basis of no molecular recombination during the expansion, (equilibrium concentrations of HF would not be significantly different), are shown in Fig. 1.

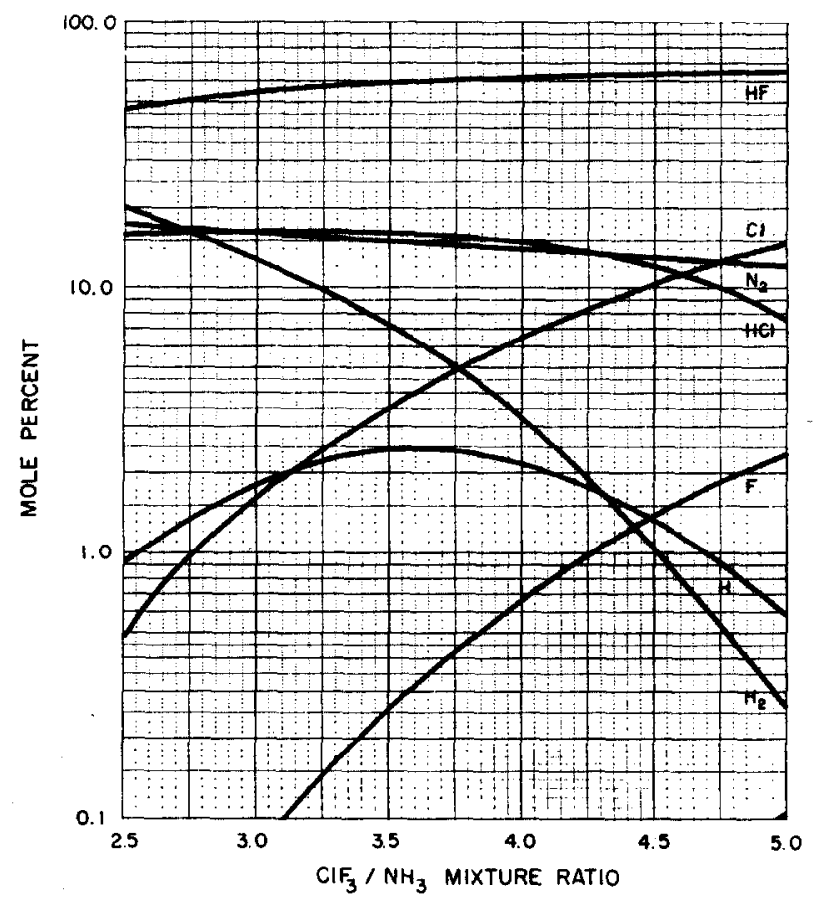

FIG. 1. Exhaust compositions determined from thermochemical calculation.

Figure 2 shows the dimensions of the interior of the motor and the location of the radiometric field of view in the exhaust jet. The flow in the conical part of the nozzle was assumed to be source-like ${ }^{(13)}$; isotherms were therefore taken to be spherical surfaces orthogonal to the diverging streamlines. Neglecting the thin boundary layer along the nozzle wall, one-dimensional calculations of the properties of the expanding gases indicated that the divergence in the flow would result in a gradient of the order of $10^{\circ} \mathrm{K}$ in the plane of the exit. This spatial variation in temperature corresponded to a local radiance within the overall experimental error of the radiometric measurements and was considered negligible; pressure and density gradients in the exit plane were likewise unimportant. Since the strength of the compression shock wave and the thickness of the mixing region in the exhaust both approach zero at the nozzle exit, and since unsteadiness of flow was considered to be of minor influcnce, the following approximation was made. 


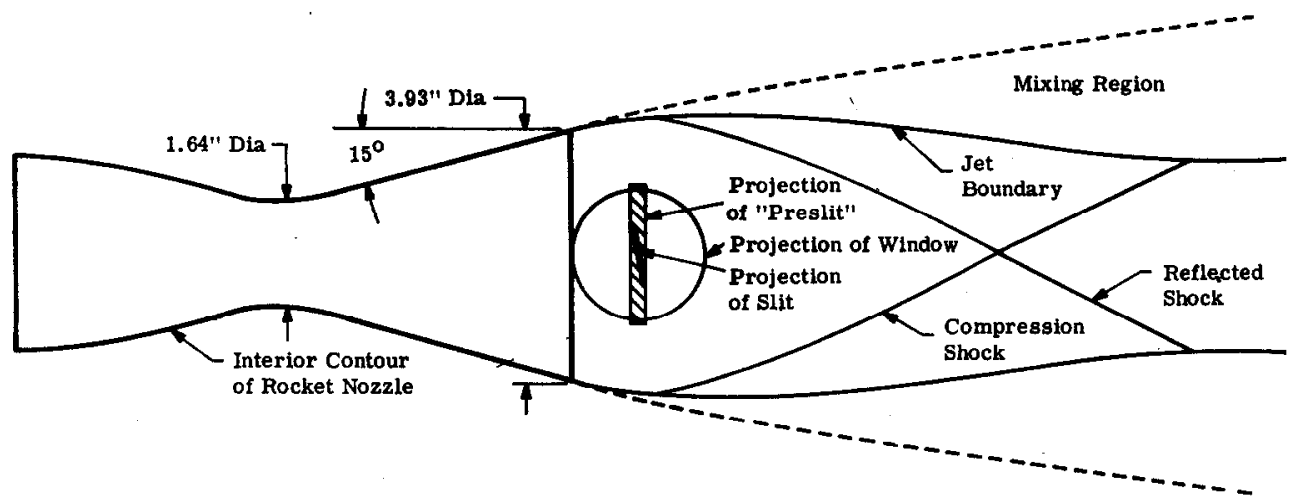

FIG. 2. Field of view in exhaust jet.

The optical path was presumed to be through a uniform isothermal region, of leng equal to the nozzle exit diameter, with a pressure and composition equal to the theoretic values for frozen flow.

\section{RADIOMETRIC INSTR UMENTATION}

The spectral radiometer used in the present investigation is described in detail els where. (14) In essence, it consists of a commercially manufactured monochromat equipped with a $\mathrm{CaF}_{2}$ prism, a set of entrance optics of special design, a blackbor radiator to provide an intensity calibration, detectors with their associated electronic and a direct-writing oscillograph. The instrument was specifically designed for its prese use in remote operation. The scanning rate, recorder chart speed, slit openings, electric gains, and time constant of the recorder input circuit were adjusted as required to produ an optimum record during the limited duration of the rocket firing; the proper setting found by experience, depended to a large degree on the particular objectives of a givt test.

Intensity calibrations were obtained by means of the blackbody source built into tl instrument and operated at temperatures spanning that in the exhaust gas. The beam fro: the source filled the entrance optics and formed an image that filled the entrance slit , the monochromator. An examination of the optical circuit ${ }^{(14)}$ would show that the indic: tions of the instrument can be reduced to the spectral radiance of an extended sourc so long as the field of view is filled.

A wavelength calibration was initially obtained from the identification of lines in th near infrared emission spectrum of $\mathrm{Hg}$ and in the absorption spectra of $\mathrm{H}_{2} \mathrm{O}, \mathrm{CO}_{2}$, an $\mathrm{HCl}$. However, the final calibration in the $2.7 \mu$ region was obtained from the spectr: measurements of HF emission; the rotational lines in the fundamental band were easi] identified and their accurately known wavelengths ${ }^{(15)}$ assigned.

The experimental arrangement by which the emission and absorption measuremen were made is shown schematically in Fig. 3. Attenuation by atmospheric $\mathrm{H}_{2} \mathrm{O}$ and $\mathrm{CC}$ was reduced to the point of being unobservable by flushing the entire optical pat $(\approx 5 \mathrm{~m})$ with extremely dry nitrogen. The absorption measurements made use of 
"greybody" source which is identical in construction to the blackbody calibration source (16) except for the radiation element. For this application, a solid graphite element of rectangular cross section was used; the effective radiating surface of 1 in. $\times 1 \mathrm{in}$. filled the field of view of the spectrometer. With this arrangement, emission-absorption measurements require three spectral scans: two during the motor operation, and one immediately before or after, respectively representing the exhaust radiance, the radiance of the greybody seen through the exhaust, and the radiance of the greybody alone. However, most of the actual data-gathering runs in the present study included additional scans: repeat scans of the flame to verify the stability of the motor operation throughout the

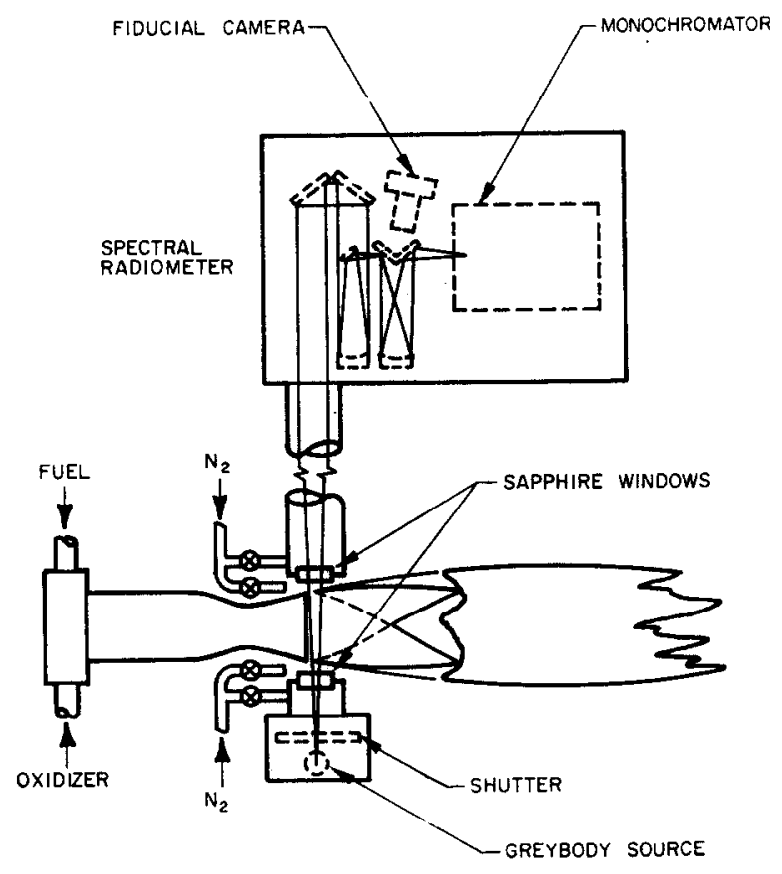

Fig. 3. Experimental arrangement used for emission-absorption measurements.

test, and scans of the greybody both before and after the run to confirm the constancy of the source temperature and the window transmittance. For the latter purpose, an auxiliary section of duct was added and flushed. In addition, immediately after each run, calibration scans were made with the blackbody set at intensities spanning those noted during the run.

\section{METHODS OF DATA REDUCTION}

The oscillograms obtained for the flame emission were reduced to absolute determinations of spectral radiance as follows. For each run, a curve of inverse sensitivity (the ratio of the deflection on the oscillogram to the spectral radiance producing it) was aetermined from the blackbody calibration spectra throughout the wavelength region of 
interest. The ability to produce these curves is contingent upon the linear response of the entire system to radiant input power. From such a curve and the original oscillogram, the values of the spectral radiance of the exhaust werc directly determined by using the relation

$$
N_{\lambda}=D_{\lambda}(N / D)_{\lambda}
$$

where $N_{\lambda}$ is the radiance corresponding to the oscillograph deflection $D_{\lambda}$, and $(N / D)_{\lambda}$ is the inverse sensitivity. Radiances were so determined at enough points to reproduce the actual shape of the emission spectrum in the original record. The values of spectral radiance were deduced without correction for the window transmission; since the attenuation was due primarily to the Fresnel losses, the reflected radiation from the opposite window would add to the exhaust radiance and, for optically thin flames, very nearly compensate for the loss through the nearer window; the residual error would be well within the overall experimental error.

In principle, the spectral emissivity as a function of wavelength can be calculated point by point from the oscillogram deflections, which are linear functions of the radiance, by the relation

$$
\epsilon(\lambda)=1-\left(D_{\lambda}^{\prime}-D_{\lambda}\right) / D_{\lambda}^{\prime \prime}
$$

where $D_{\lambda}, D_{\lambda}^{\prime}$, and $D_{\lambda}^{\prime \prime}$, respectively, are the deflections corresponding to the exhaust radiance, the radiance of the greybody viewed through the flame, and the actual greybody radiance. However, this calculation becomes very inaccurate in regions of low emission and absorption. Therefore, the emissivities reported herein were determined by using a different procedure. The effective gas temperature $T$ was deduced from the observed emission and absorption in the most prominent lines using the definition

$$
N_{\lambda}^{*}(T)=N_{\lambda, i} / \epsilon\left(\lambda_{i}\right)
$$

where $N_{\lambda}^{*}(T)$ is the equivalent spectral radiance of a blackbody at temperature $T$, and the spectral radiance and emissivity at particular wavelengths $\lambda_{i}$ were determined by equations (1) and (2). The fact that such absorption-emission temperature determinations are independent of instrument resolution can be shown. (17) In the present study an average was taken of the temperatures obtained for lines $P(5)$ through $R(4)$, which were most easily seen in absorption against the background of greybody emission. The spectral emissivities throughout the band were then calculated from

$$
\epsilon(\lambda)=N_{\lambda} / N_{\lambda}^{*}(T) \text {. }
$$

It should be noted that the spectral emissivities and radiances so determined are not "absolute" in the sense of being independent of the instrumental settings. Rather, they represent effective averages for the resolution at which they were obtained; conversion to "absolute" values would require precise knowledge of the spectrometer slit function. However, they may be properly called "absolute" in the sense of providing the basis for determining the actual amount of radiant power emitted by the gas; the integrated value of these effective spectral quantities over the entire band, or part of the band encompassing several lines, would be independent of the spectrometer slit opening.

It should also be noted that knowledge of the greybody temperature is not required for the determination of flame emissivity, and that equation (2) is applicable even when 
the source temperature is lower than the flame temperature. However, the best results are obtained when the source is considerably brighter than the flame throughout the entire spectral region of interest.

\section{EMISSION AND ABSORPTION SPECTRA}

Figure 4 is an unretouched reproduction of an original recording of the flame emission obtained at a spectral slit width of $0.003 \mu$ using the $\mathrm{PbS}$ detector. The spectrum is primarily that of HF in the fundamental vibration rotation band. The lines in the $R$-branch are resolved out to $R(11)$, beyond which they merge to form a band head at $2.27 \mu$; the lines in the $P$-branch are recognizable out to $P(14)$, beyond which the sensiti-

TABLE 1. SumMaRY OF DIRECTLY DETERMINED EMISSIVITIES AND TEMPERATURES

\begin{tabular}{|c|c|c|c|c|c|c|c|c|}
\hline \multirow[t]{3}{*}{$\begin{array}{l}\text { Wavelcngth } \\
\qquad(\mu)\end{array}$} & \multicolumn{8}{|c|}{ Oxidizer/Fuel Mixture Ratio } \\
\hline & \multicolumn{2}{|c|}{$3 \cdot 27$} & \multicolumn{2}{|c|}{$3 \cdot 49$} & \multicolumn{2}{|c|}{$3 \cdot 75$} & \multicolumn{2}{|c|}{$3 \cdot 65$} \\
\hline & $\epsilon(\lambda)$ & $T$ & $\epsilon(\lambda)$ & $T$ &,$\in(\lambda)$ & $T$ & $\epsilon(\lambda)$ & $T$ \\
\hline $\begin{array}{l}2.673 P(5) \\
2.640 P(4) \\
2.608 P(3) \\
2.579 P(2) \\
2.551 P(1) \\
2.499 R(0) \\
2.476 R(1) \\
2.454 R(2) \\
2.433 R(3) \\
2.414 R(4)\end{array}$ & $\begin{array}{l}0.206 \\
0.129 \\
0.142 \\
0.103 \\
0.096 \\
0.098 \\
0.092 \\
0.112 \\
0.126 \\
0.103\end{array}$ & $\begin{array}{l}1174 \\
1263 \\
1173 \\
1194 \\
1170 \\
1192 \\
1209 \\
1242 \\
1218 \\
1258\end{array}$ & $\begin{array}{l}0.191 \\
0.141 \\
0.133 \\
0.106 \\
0.084 \\
0.094 \\
0.102 \\
0.110 \\
0.108 \\
0.114\end{array}$ & $\begin{array}{l}1257 \\
1311 \\
1287 \\
1281 \\
1259 \\
1264 \\
1251 \\
1286 \\
1318 \\
1311\end{array}$ & $\begin{array}{l}0.191 \\
.0 \cdot 122 \\
0 \cdot 179 \\
0 \cdot 144 \\
0.087 \\
0 \cdot 104 \\
0 \cdot 113 \\
0 \cdot 108 \\
0 \cdot 109 \\
0 \cdot 107\end{array}$ & $\begin{array}{l}1211 \\
1301 \\
1150 \\
1131 \\
1163 \\
1195 \\
1182 \\
1292 \\
1281 \\
1268\end{array}$ & $\begin{array}{l}0.220 \\
0.166 \\
0.195 \\
0.126 \\
0.105 \\
0.083 \\
0.125 \\
0.148 \\
0.157 \\
0.133\end{array}$ & $\begin{array}{l}1183 \\
1263 \\
1203 \\
1244 \\
1238 \\
1236 \\
1252 \\
1288 \\
1288 \\
1288\end{array}$ \\
\hline $\begin{array}{l}\text { mean } \\
\text { average } \\
\text { deviation }\end{array}$ & & 1209 & & $\begin{array}{r}1283 \\
\pm 20\end{array}$ & & 1217 & & 1248 \\
\hline
\end{tabular}

vity of the PbS detector falls off rapidly. The actual line-widths are much smaller than apparent in the original recording; the observed triangular line shape is due to geometrical effects in the monochromator and is more representative of the slit-function of the instrument than of the true line shape. In addition to the rotational lines in the $1 \rightarrow 0$ fundamental band, a number of lines in the $2 \rightarrow 1$ band were identified, and a few in the $3 \rightarrow 2$ band were tentatively identified, as indicated. A typical set of unreduced absorption and emission spectra are shown in Fig. 5; the requirement for additional scans necessitated the somewhat lower resolution. The lines identified both in emission and absorption are indicated.

Since combustion in a liquid propellant rocket engine will not remain stable over a very wide range of fuel-oxidizer mixture ratio, the combustion temperature and hence the exhaust temperature (the ratio of which is nearly constant for a De Laval nozzle of 


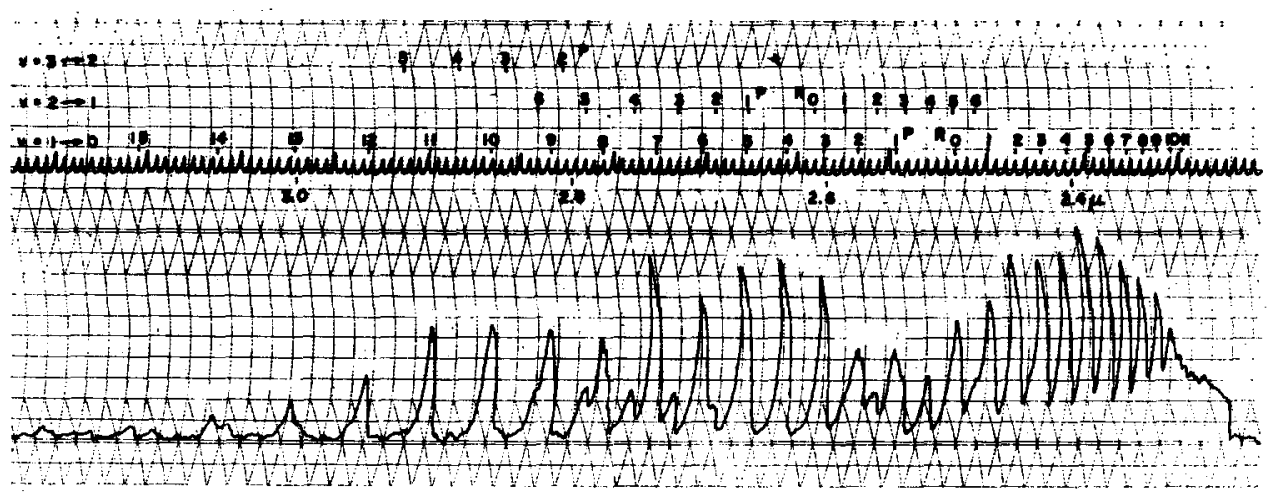

Fig. 4. Emission spectrum of $\mathrm{HF}$ in the $2 \cdot 5 \mu$ region.

fixed geometry) could not be varied appreciably during the present study. In general, slightly greater radiances were observed with leaner mixtures, but the resultant differences in the spectral emissivities, to be discussed presently, were not significant. The spectra presented in Figs. 4 and 5 are, however, typical of many measurements which were made over a limited range of mixture ratios, and which were without exception comparable in magnitude and nearly identical in spectral detail.

\section{SPECTRAL RADIANCES AND EMISSIVITIES}

Using equation (1), the emission spectrum can be reduced to spectral radiance as a function of wavelength. The radiance spectra can be re-expressed in terms of spectral emissivities by use of the emission and absorption measurements in equations (2), (3), and (4). In essence, this procedure is a normalization of the measured radiances using
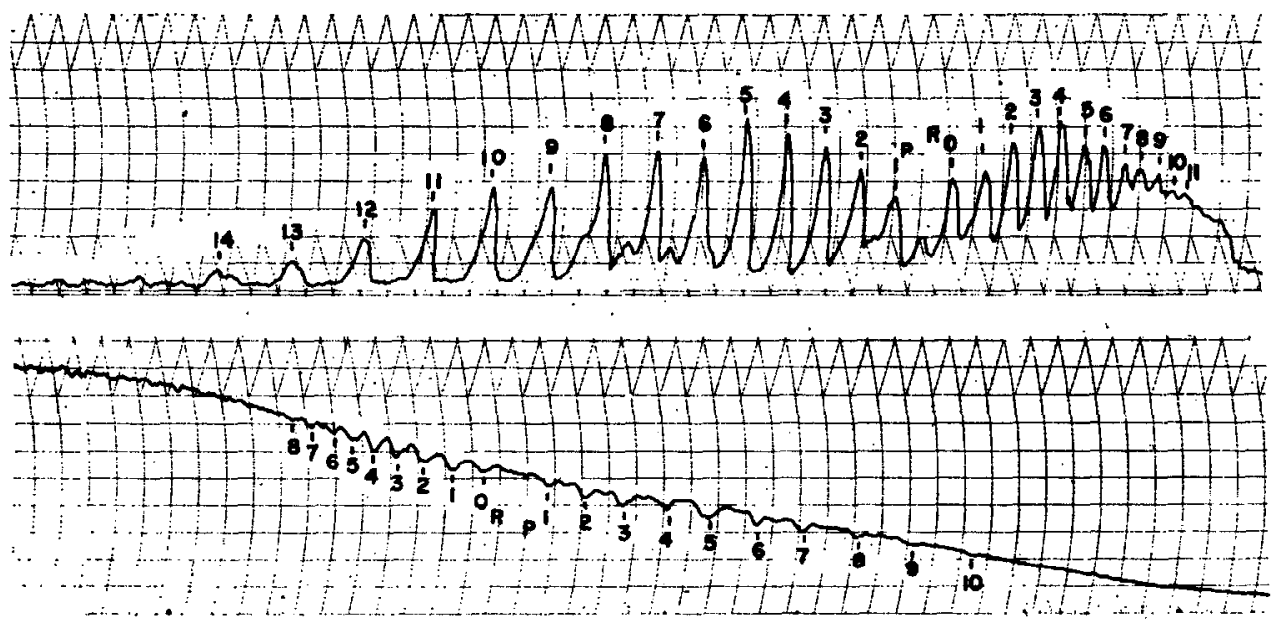

FIG. 5. Typical unreduced emission and absorption spectra. 
(a)

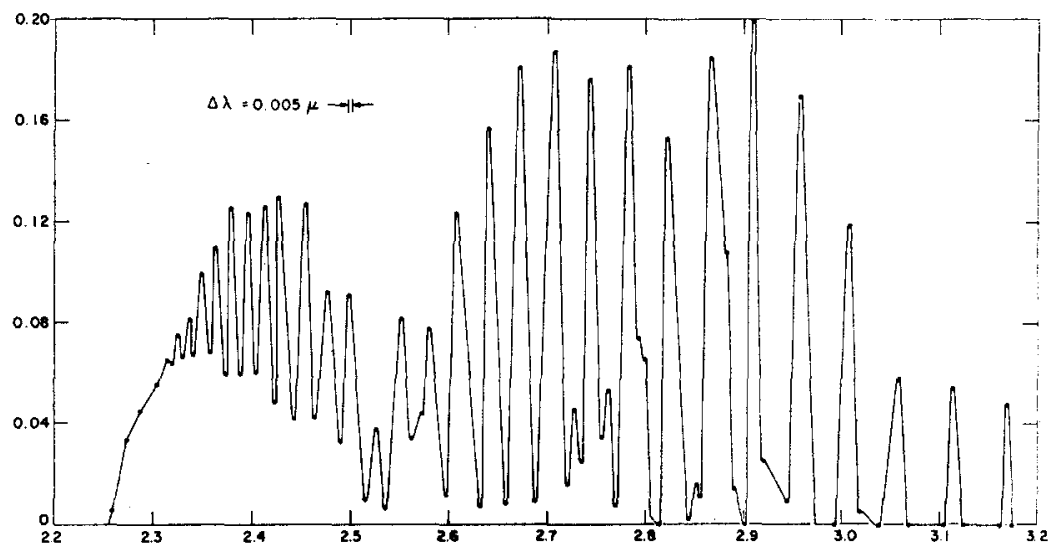

(b)

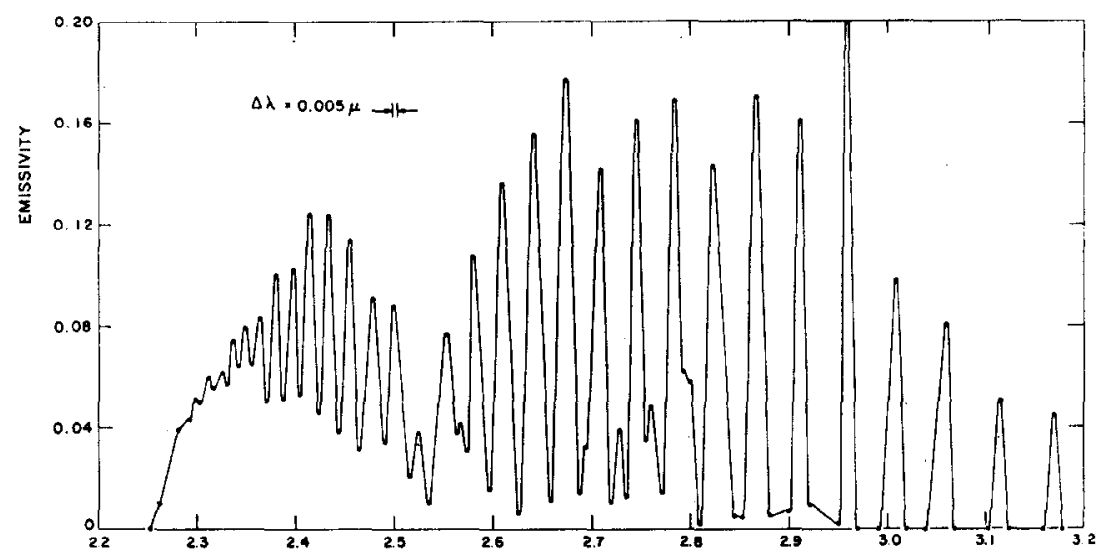

(c)

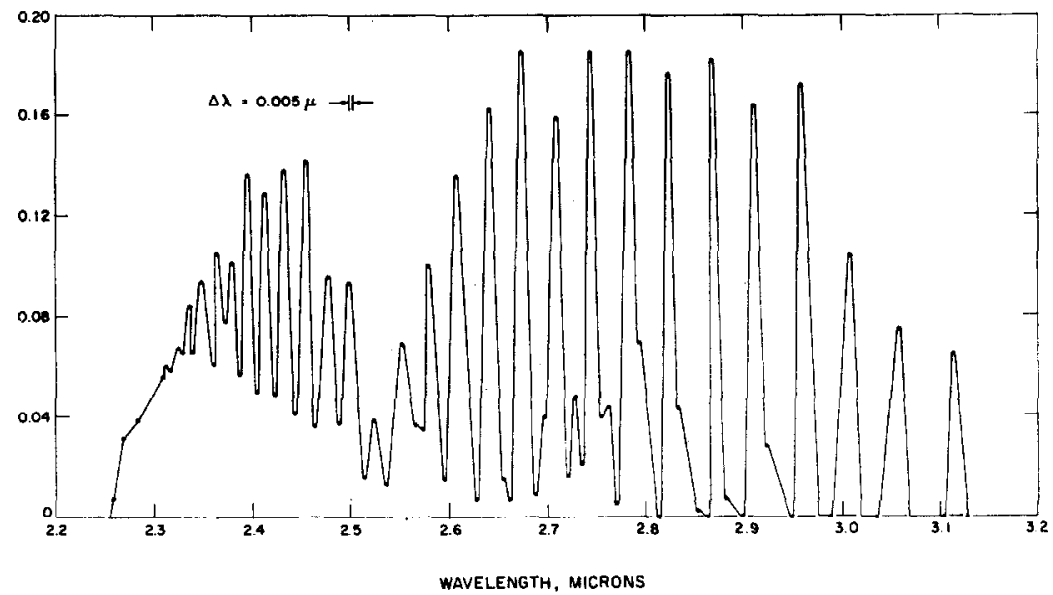

FIG. 6. Spectral emissivity of HF at various mixture ratios: (a) 3.27 ; (b) 3.49 ; (c) 3.75 . 
Infrared spectral radiances and emissivities of hydrogen fluoride

(a)

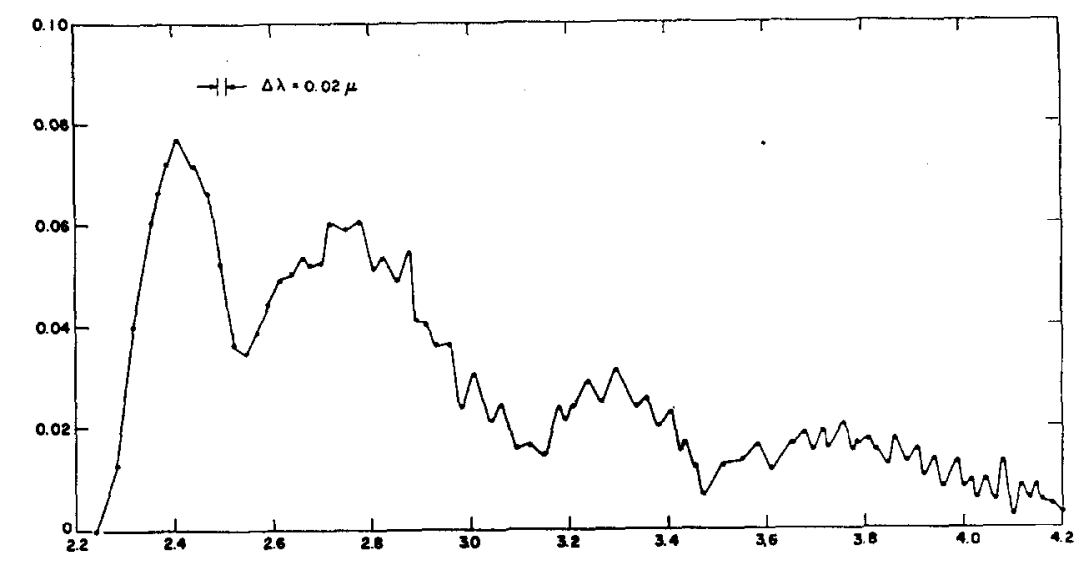

(b)

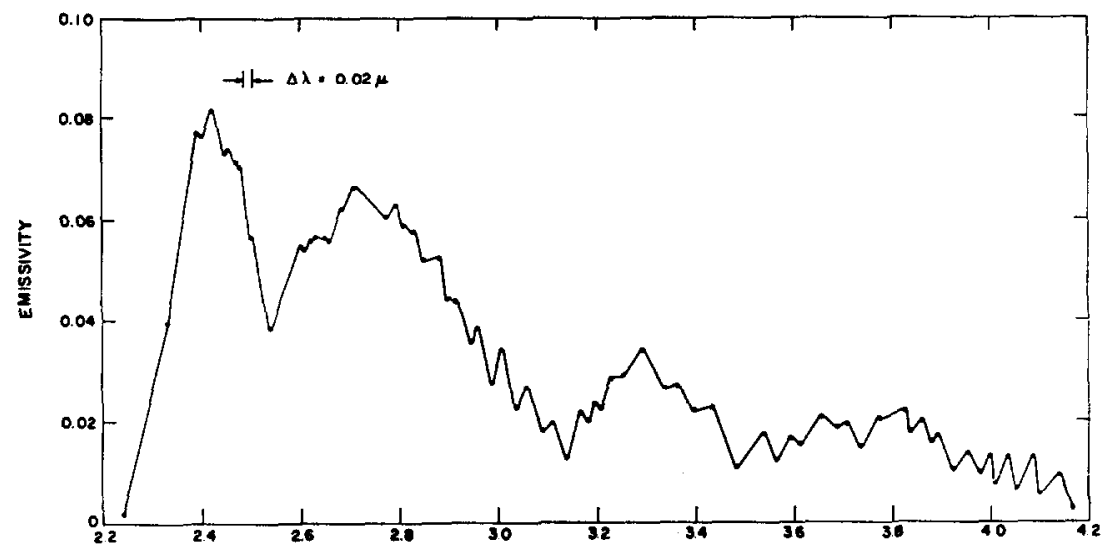

(c)

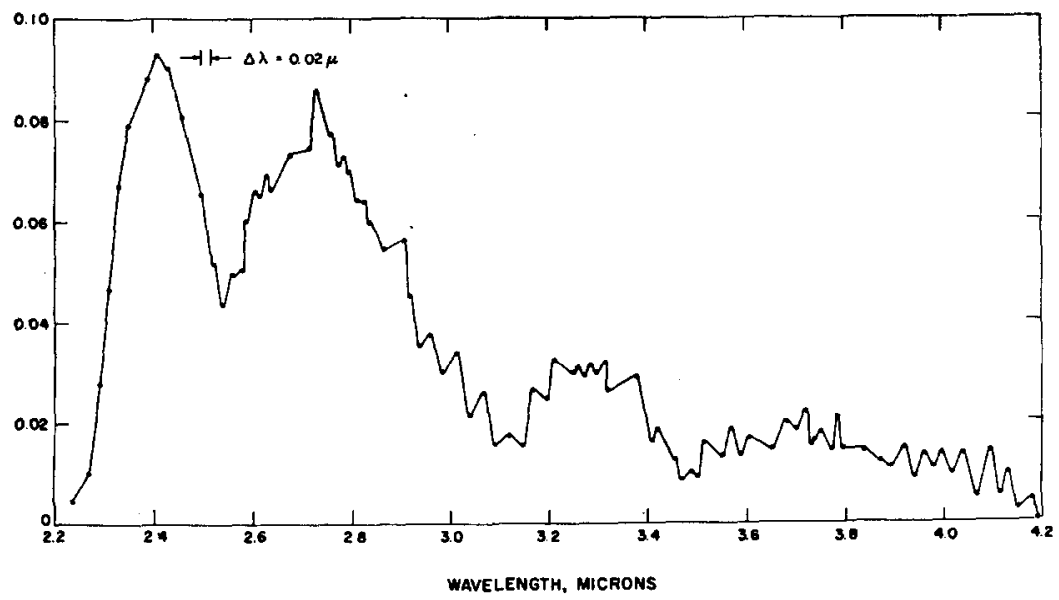

FIG. 7. Spectral emissivity of $\mathrm{HF}$ and $\mathrm{HCl}$ at various mixture ratios: (a) 3.65 ; (b) 3.74 ; (c) 3.94 . 
the appropriate values of the blackbody function corresponding to the temperature assigned to the gas. As mentioned previously, the lines $P(5)$ through $R(4)$ were most easily measured in absorption as well as in emission; the emissivities and gas temperatures deduced from these measurements using equations (2) and (3) are summarized in Table 1. The normalized radiances, i.e. spectral emissivities computed by equation (4), are presented in Fig. 6. The overall similarities in the spectra are mainly a consequence of the fact that the concentration of HF, the major species in the exhaust, is not very sensitive to the mixture ratio. The dissimilarities in the relative amplitudes among the more intense lines from one spectrum to another were attributed to slight fluctuations in gas temperature during scanning; even repeat scans during the same run showed such differences.

In addition to the measurements using a $\mathrm{PbS}$ detector, spectra were obtained with a $\mathrm{PbSe}$ detector for the purpose of observing the longer-wavelength emission from $\mathrm{HCl}$,

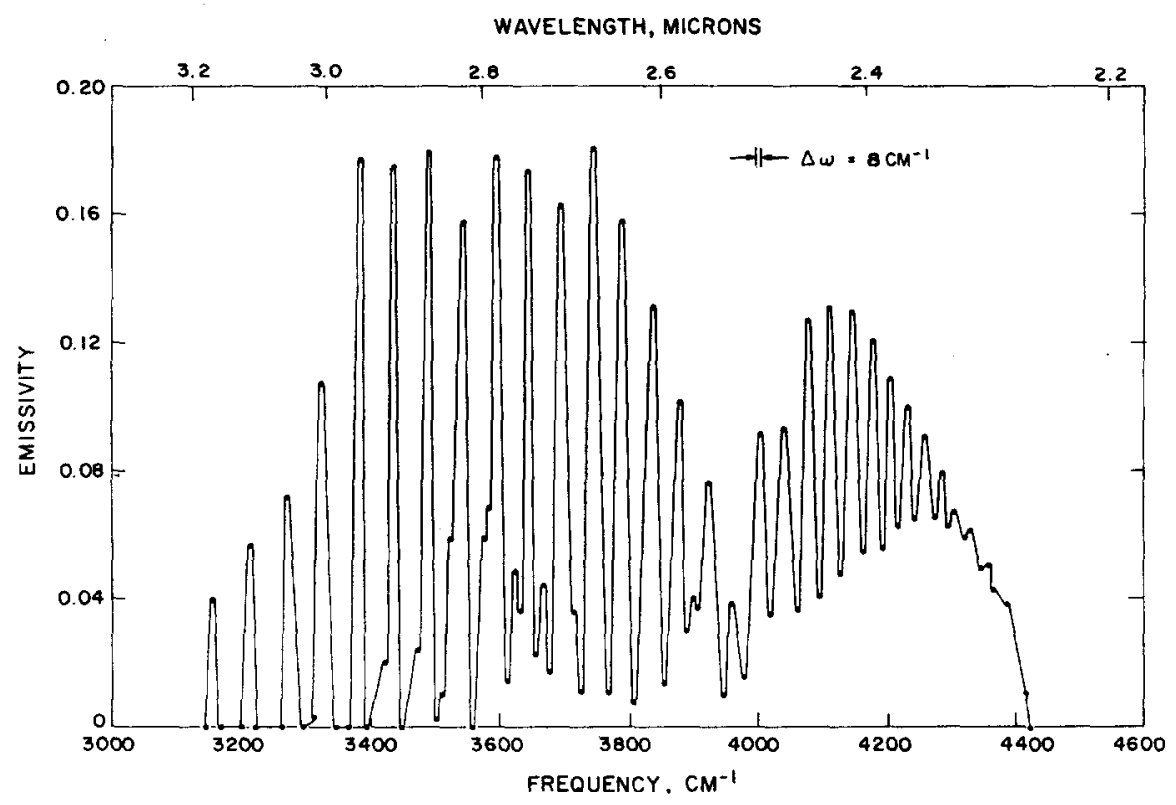

FIG. 8. Spectral emissivity of $\mathrm{HF}: T \approx 1250^{\circ} \mathrm{K}, p \approx 0.5 \mathrm{~atm}, L=10 \mathrm{~cm}, X(\mathrm{HF}) \approx 3 \mathrm{~cm}$-atm.

the other infrared emitting species in the exhaust from $\mathrm{ClF}_{3}-\mathrm{NH}_{3}$ combustion. Because the $\mathrm{PbSe}$ cell has a lower detectivity, the emission spectra were necessarily obtained at a considerably lower resolution, and it was not possible to measure absorption. Therefore, emissivities were calculated by using equation (4) with an assumed gas temperature consistent with that previously determincd by the $\mathrm{PbS}$ measurements. Figure 7 shows typical spectra of the emissivity of $\mathrm{HF}$ and $\mathrm{HCl}$ for various mixture ratios. Because of the lower resolution, there is comparatively little spectral detail, although both branches of the $\mathrm{HCl}$ fundamental band, centered at approximately $3.5 \mu$, are evident. Also apparent is the slight overlap of the $R$-branch of $\mathrm{HCl}$ with the $P$-branch of $\mathrm{HF}$ in the $3 \cdot 0$ to $3 \cdot 2 \mu$ region. 
Average spectral emissivities are presented in Figs. 8 and 9 , which are composites of Figs. 6 and 7, respectively. These presentations are considered to be representative of a gas temperature of $1250^{\circ} \mathrm{K}$ and optical depths of $3 \mathrm{~cm}$-atm for $\mathrm{HF}$ and $1 \mathrm{~cm}$-atm for $\mathrm{HCl}$.

The spectral radiances and emissivities reported herein appear to be representative, in overall magnitudes, of gases in thermal equilibrium at the assigned temperatures and optical depths. However, temperature gradients, vibrational lags, partial recombination, or other nonequilibrium effects in the expansion of combustion products in a De Laval nozzle, if present, can influence the observed spectra of the supersonic exhaust gases.

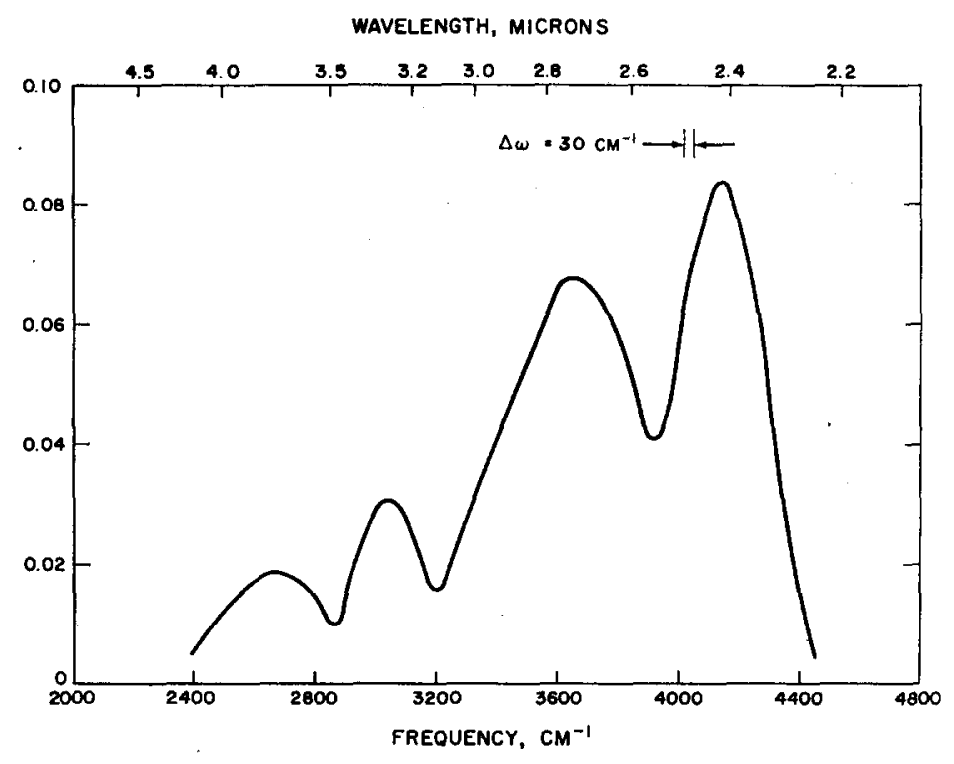

FIG. 9. Spectral emissivity of $\mathrm{HF}$ and $\mathrm{HCl}: T \approx 1250^{\circ} \mathrm{K}, p \approx 0.5 \mathrm{~atm}, L=10 \mathrm{~cm}$, $X(H F) \approx 3 \mathrm{~cm}-\mathrm{atm}, X(\mathrm{HCl}) \approx 1 \mathrm{~cm}-\mathrm{atm}$.

Some of these effects will be discussed in a forthcoming report, ${ }^{(18)}$ in which the data will be analyzed in more detail and compared with theoretical predictions. $(19,20)$

The present measurements of spectral emissivities can be used to predict radiances from similar gaseous systems, at the same temperature but for other optical depths, by well known methods based upon appropriate molecular band models. ${ }^{(21,22,23)}$ Extrapolation to systems at other temperatures is more difficult, requiring knowledge of the variation with temperature of the average spectral absorption coefficients corresponding to the measured absorptances. Therefore, additional measurements over a range of temperatures would be requisite for a completely empirical evaluation of such variation. Alternatively, theoretical results, which agree with the measurements at the observed optical depths and temperatures, can be applied elsewhere with more confidence.

Acknowledgement-Considerable credit is due Mr. J. D. ENLOE for his efforts in the operation of the rocket test facilities and Mr. Q. S. ANDERSON for his assistance in making the spectroscopic measurements. 


\section{REFERENCES}

1. R. H. Tourin, J. Opt. Soc. Amer., 51, 175 (1961).

2. D. K. Edwards, J. Opt. Soc. Amer. 50, 617 (1960).

3. U. P: OpPenheim and Y. Ben-ARYeH, J. Opt. Soc. Amer. 53, 344 (1963).

4. M. Steinberg and W. O. Davies, J. Chem. Phys., 34, 1373 (1961).

5. C. C. Ferriso, J. Chem. Phys. 37, 1955 (1962).

6. W. Malkmus, Infrared Emissivity of Carbon Dioxide, Rept. No. AE62-0204, General Dynamics. Astronautics, San Diego, California (1962).

7. R. H. Tourin, J. Opt. Soc. Amer. 51, 1225 (1961).

8. D. E. Burch and D. A. Gryvnak, Rept. No. V-1929, Ford Motor Co., Aeronutronics Division Newport Beach, California (1962).

9. C. C. FerRiso and C. B. Ludwig, High Temperature Emissivities of the $2 \cdot 7 \mu$ Band of Water Vapo: between $1000^{\circ} \mathrm{K}$ and $2200^{\circ} \mathrm{K}$. Rept. No. BBE 63-002. General Dynamics/Astronautics, San Diego California (1963).

10. D. K. EDwards, Studies of Infrared Radiation in Gases, Rept. No. 62-65, Dept. of Engineering University of California, Los Angeles, California (1963).

11. R. Eulner, J. Mertens and R. L. Potter, Comb. and Flame, 5, 1 (1961).

12. J. Q. WEBER, Private communication of rocket performance calculations. Rocketdyne, Canoga Park California.

13. G. V. R. RAO, Amer. Rocket Soc. J. 29, 606 (1959).

14. F. S. Simmons and A. G. DeBell, Spectral Radiometry and Two-Path Pyrometry of Rocket Exhaus Jets, Temperature, Vol. III, Part 2, Reinhold, New York (1963).

15. G. A. Kuipers, D. F. Smith and A. H. Nielsen, J. Chem. Phys. 25, 275 (1956).

16. F. S. Simmons, A. G. DeBell and Q. S. Anderson, Rev. Sci. Instrum. 32, 1265 (1961).

17. H. J. Babrov, J. Opt. Soc. Amer. 51, 171 (1961).

18. W. Malkmus, S. A. Golden and F. S. Simmons, A Comparison of Theoretical and Experimenta Values for the Spectral Emissivity of Hydrogen Fluoride at $1000^{\circ} \mathrm{C}$, in preparation.

19. W. MAlKmus and A. ThOMSON, JQSRT 2, 17 (1962).

20. S. A. Golden, Matrix Elements and Integrated Intensities of Vibration-Rotation Transitions in HF Rocketdyne, Canoga Park, California (1962).

21. S. S. Penner, Quantitative Molecular Spectroscopy and Gas Emissivities, pp. 317-327, Addison Wesley, New York (1959).

22. H. J. BABrov, P. M. Henry and R. H. Tourin, Methods for Predicting Infrared Radiance of Flame by Extrapolation from Laboratory Measurements, Warner and Swasey Co., Rept. AFCRL-107, (1961).

23. G. N. Plass, Methods for the Presentation of Laboratory Infrared Absorption Measurements, $\mathrm{i}$ : Advances in Molecular Spectroscopy, Pergamon Press, Oxford (1962). 